\section{A case of cerebral venous thrombosis following the use of contraceptive medication}

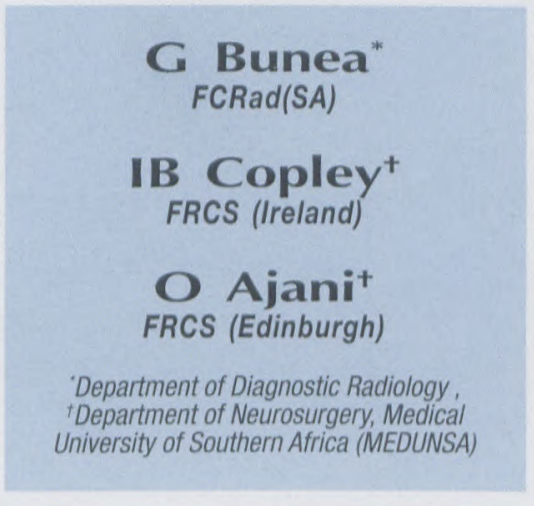

\section{Introduction}

Venous sinus thrombosis is a rare event and may have an insidious onset and late sequelae in contradistinction to cavernous sinus thrombosis associated with infection and florid signs. Thrombosis involving the dural sinuses is due to three causes: those from changes in blood flow, changes in the vessel wall, or blood abnormalities. Blood flow changes are most commonly seen in dehydration.

Changes in flow and vessel wall are seen in depressed fractures about the midline of the vault. Operative interhemispheric (e.g. transcallosal) procedures near the superior sagittal sinus may produce local trauma and stasis. Another entity is the vasculitis of Behçet's disease. Common, non-infectious causes of cerebral venous thrombosis include oral contraceptives, pregnancy and the puerperium. Abnormal changes in blood constituents are seen in malnutrition, and blood deficiencies antithrombin III, protein S \& C, disseminated intravascular coagulation, iron deficiency anaemia. ${ }^{1}$

Occlusion of the anterior third of the superior sagittal sinus does not produce symptoms or signs whereas thrombosis of the middle third produces upper motor neurone signs, hemi- or quadriparesis. Visual field disturbances or blindness may occur with occlusion of the posterior third of the sinus rapidly followed by decreasing level of consciousness.

Thrombosis of a transverse sinus is less significant than if the opposite sinus is also occluded. Any involvement of the posterior two-thirds is associated with cerebral oedema indicated by engorgement of retinal veins, 


\section{A case of cerebral venous thrombosis following the use of contraceptive medication}

\section{from page 33}

meningism and decreased level of consciousness. In subdural parafalx empyema, venous thrombosis followed by infarction may be seen in neglected cases. As the empyema may be restricted to one side or another of the falx, so the neurological deficit may be unilateral before rapid deterioration as the whole superior sagittal sinus becomes involved.

\section{Case report}

The patient P.M., a healthy 23 yearold woman, had no previous medical history. She had a child of 5 years of age. A year after delivery, she began with three-monthly injections of norethisterone enantate $200 \mathrm{mg}$. Soon after starting on this treatment, she developed headaches and amenorrhea. Three months prior to admission, owing to the headaches, she was changed to levonorgestrel $150 \mu \mathrm{g}$, ethinyl oestradiol $30 \mu \mathrm{g}$ oral contraceptive. The change relieved the headaches and there were scanty periods. Approximately two days prior to admission, the patient presented at a peripheral hospital with severe headache, nausea and vomiting, decreased vision and confusion (GCS 13/15) followed by deepening coma. On recovering consciousness, she had a right hemiparesis. Her mental recovery was such as to provide the foregoing history. Computerized tomography (CT) done at the peripheral hospital (Figure 1 and 2) was reported as "a large subdural haematoma (possible venous sinus) occipitally; blood in the quadrigeminal cisterns, possible subarachnoid haemorrhage (SAH), blood in the region of the straight sinus. Post contrast filling defect in the sagittal sinus - possible dural sinus thrombosis". Because of the reported $\mathrm{SAH}$, the patient was treated with nimodipine. Four-vessel angiography,
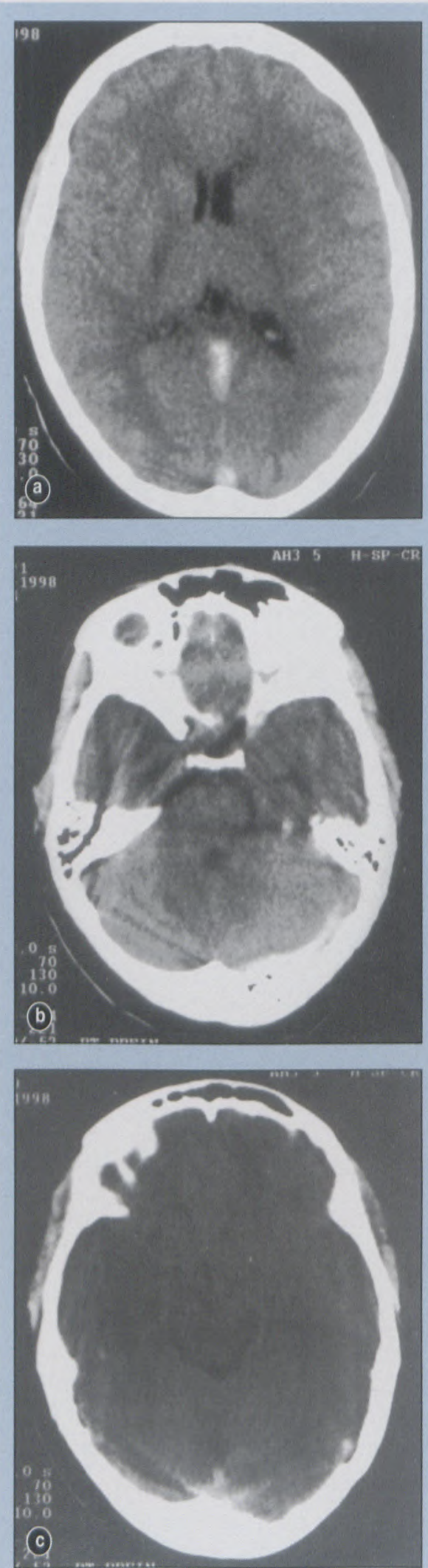

Figure $1 a, b$ and $c$ : CT scan pre-contrast on admission demonstrated high-density thrombus in straight sinus, superior sagittal sinus, and in left transverse sinus. A thrombosed vein (cord sign) is seen on the medial aspect of the left temporal lobe

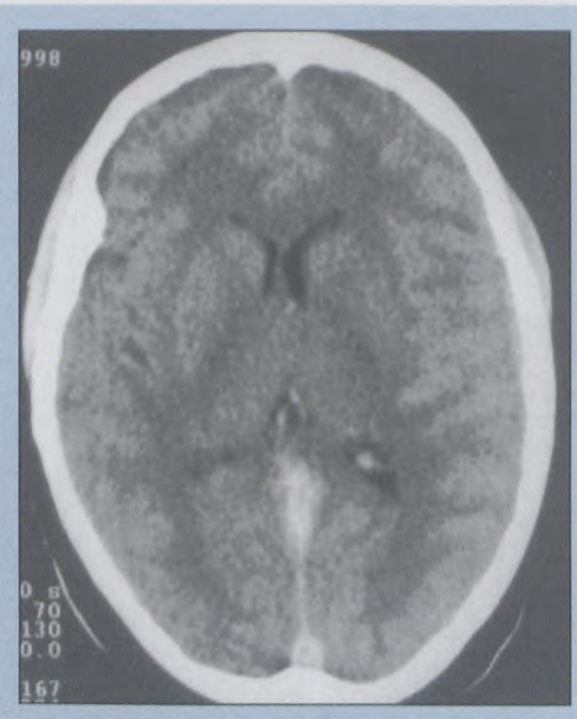

Figure 2: CT scan post-contrast shows the empty delta sign in the superior sagittal sinus
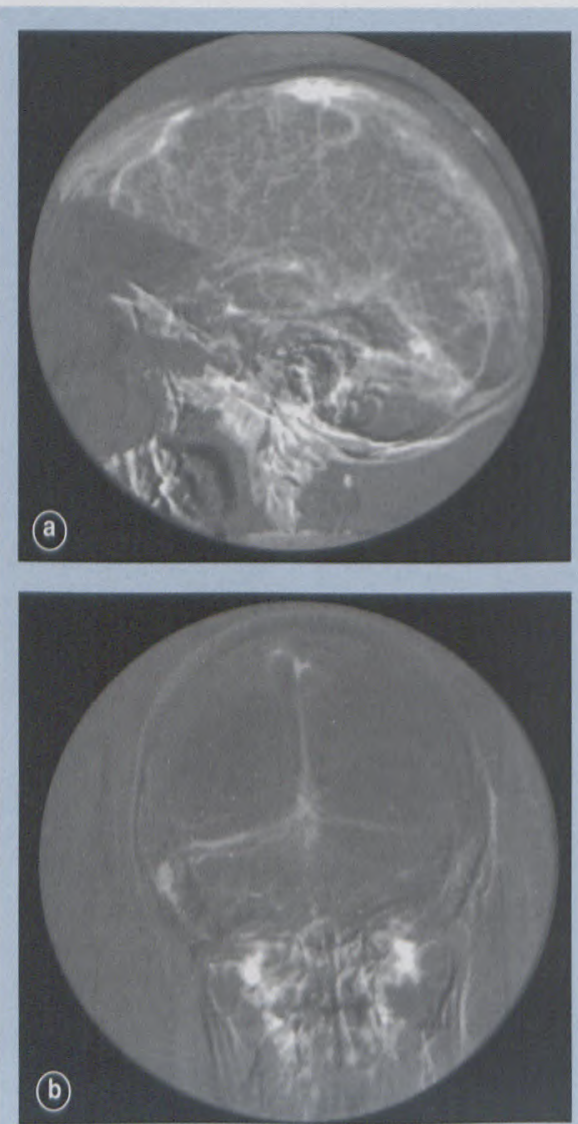

Figure $3 a$ and $b$ : Digital cerebral angiogram in the venous phase demonstrated poor and irregular filling of the superior sagittal sinus, straight sinus, vein of Galen, and transverse sinuses. Note prominent cortical tributary veins draining into the rostral aspect of the superior sagittal sinus

performed 11 days later (Figure 3), confirmed the diagnosis of venous sinus thrombosis. 


\section{A case of cerebral venous thrombosis}

following the use of contraceptive medication

\section{from page 34}
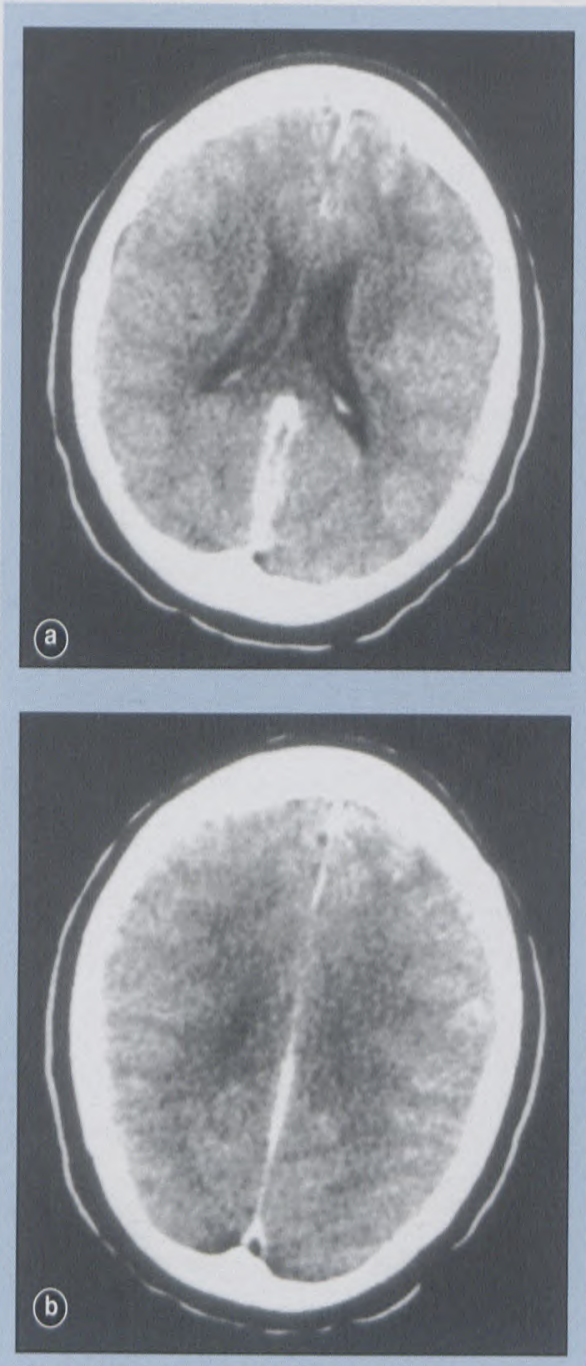

Figure $4 a$ and $b$ : Repeat CT scan with contrast injection, 12 days later showed strong enhancement of the straight sinus with a central filling defect, and the empty delta sign in the superior sagittal sinus

A second CT with contrast (Figure 4) was done 12 days later which showed a clear delta sign and clot within straight sinus. Magnetic resonance (MR) imaging was requested at 18 days and showed typical high signal thrombus (Figure 5) in the dural sinuses. It also showed a left cortical venous infarct.

\section{Discussion}

The cerebral side effects of the contraceptive medication include nonspecific headaches or migraine, withdrawal headaches, benign intracranial
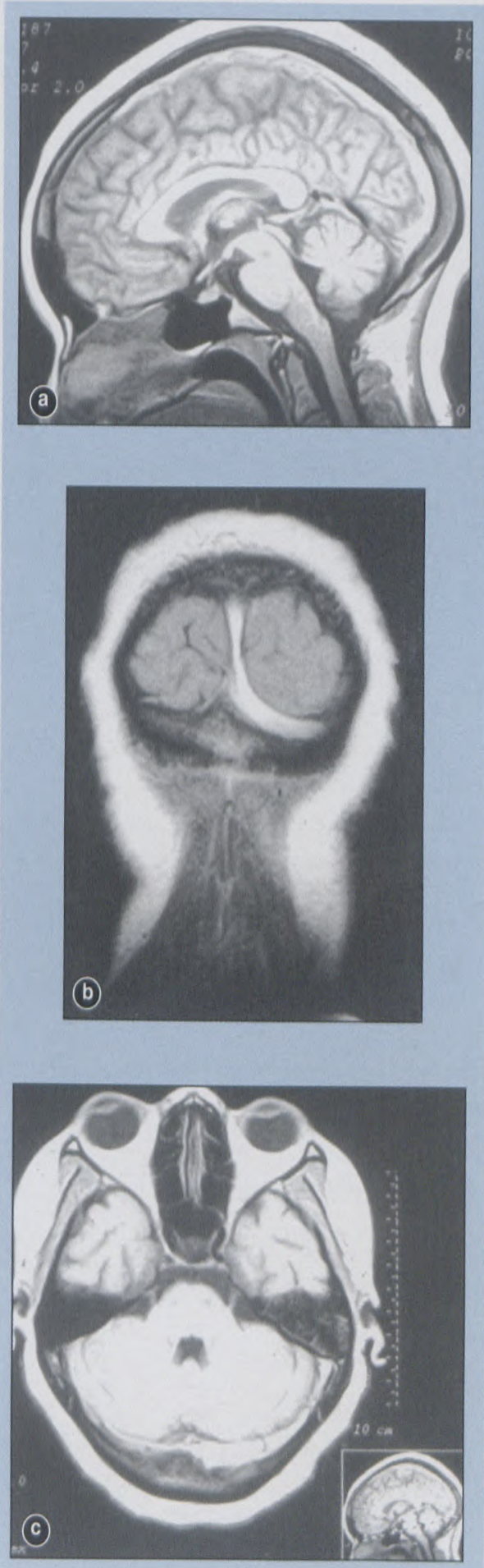

hypertension, and cerebral venous thrombosis (CVT). The evaluation of a patient on contraceptive medication, who complains of worsening headaches, requires exclusion of $\mathrm{CVT}^{2}$ by computed tomography or magnetic resonance imaging.

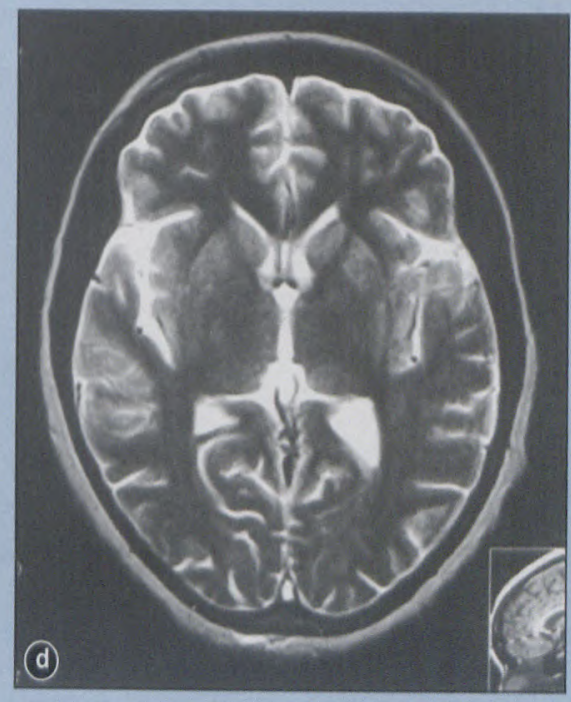

Figure 5a, b, c and d: Sagittal, coronal, and axial T1 and T2-weighted MR images showed high intensity signal within the superior sagittal sinus

The manufacturers state that the occurrence for the first time of a migraine type of headache, the more frequent occurrence of an unusually severe headache, or sudden perception disorders is sufficient reason for immediate discontinuation. ${ }^{3} \mathrm{~A}$ mere change of the drug is probably hazardous. This case shows that CVT can masquerade for many months as a simple headache or even a benign intracranial hypertension.

Extensions to the deep cerebral veins are associated with a sudden neurological deterioration and poor outcome. Imaging is critical to the diagnosis of this disorder, which can be made by non-invasive modalities such as CT or MR.

Non-enhanced CT scans may show hyperdense thrombus in the dural sinus (dense sinus sign), deep cerebral veins, or cortical veins (cord sign), cortical and subcortical haemorrhagic infarctions, and diffuse cerebral swelling. After contrast the thrombosed sinus remains unopacified with enhancement of the 


\section{from page 35}

collaterals in the dura leaves (empty delta sign), the falx appears thickened, there is tentorial (shaggy tentorium) and gyral enhancement, and the transcortical medullary veins may enhance strongly. Deep cerebral vein thrombosis appears as a high density thrombus in the deep veins, vein of Galen, or straight sinus, with or without basal ganglia infarctions and petechial haemorrhages. The differential diagnosis of dural sinus thrombosis on CT scans includes normal neonates with unmyelinated brain and dense sinus, high-splitting tentorium and pseudodelta sign seen in subarachnoid haemorrhages.

MR findings vary with clot age. An acute thrombus is iso-intense with the cortex on Tl-weighted images, older haematomas are hyper-intense on T1weighted scans and hypo-intense on T2-weighted images, while sub-acute thrombi are typically hyper-intense on all pulse sequences. In the chronic phase, prominent collateral venous channels can be seen around and within the thrombosed sinus (i.e. recanalisation), and intense enhancement of the thrombus after gadolinium injection (i.e. conversion to vascularized connective tissue). Other causes of increased signal within a sinus on spin-echo images must be excluded, such as: turbulent or slow flow, flow entry phenomenon, even echo rephasing, and flow compensation techniques. Flow enhancing gradient-echo sequences along with spinecho sequence should allow the differentiation. In acute thrombosis, high field strengths can give a low signal which can be confused with a patent dural sinus. MR Angiography or Venography provides conclusive evidence of flow in the sinus rather than relying on flow-related enhancement effects in standard imaging.

Angiographic signs of a thrombosed sinus include non-filling of the dural sinus, filling defects, enlarged medullary veins and other collaterals. Thrombosed cortical veins are seen as contrast collections which appear to hang in space with contrast persisting well into the very late venous phase. Deep cerebral vein thrombosis is seen as the non-filling of the vein of Galen and internal cerebral veins, with or without collaterals. ${ }^{4}$

Cerebral CTVenography, possible with spiral CT and three-dimensional reconstructions, allows an accurate evaluation of the flow in the cerebral venous system. ${ }^{5}$ Venous transcranial Doppler ultrasonography can be used as a monitoring tool in the evaluation of the collateral venous flow in superior sagittal sinus thrombosis, however it needs further evaluation. ${ }^{6}$ Apart from anticoagulation and control of raised intracranial pressure, selective venous administrations of fibrinolytic agents by an interventional neuroradiologist is possible. ${ }^{7}$ It is conjectural as to whether or not the use of nimodipine contributed to the patient's eventual excellent recovery.

\section{References}

1. Hoffman MW, Bill PLA, Bhigjee AI, Modi G, Haribhai HC, Keble C. The clinicoradiological profile of cerebral venous thrombosis. $S \mathrm{Afr}$ Med. J,1992: 82: 341-348.

2. Naim-Ur-Rahman, Abdul Tahman Al Tahan. Computed tomographic evidence of an extensive thrombosis and infarction of the deep venous system. Stroke, 1993: 24,5: 744-746.

3. MIMS Medical Specialties, 1998: 38,4: 184.

4. Osborn AG. Diagnostic neuroradiology. St Louis : Mosby. 1994 : 385-395.

5. Casey SO, Alberico RA, Patel M, Jiminez JM, et al. Cerebral CT venography. Radiology, 1996: 198,1: 163-170.

6. Valdueza JM, Schultz M, Harms L, Einhäupe KM. Venous transcranial Doppler, ultrasound monitoring in acute dural sinus thrombosis. Stroke, 1995: 26,7: 1196-9.

7. Scott JA, Pascuzzi RM, Hall PV, Becker GJ Treatment of dural sinus thrombosis with local urokinase infusion. JNeurosurg, 1988: 68: 284 . 287. 